\title{
The free topological group on a cell complex
}

\section{J.P.L. Hardy}

It is proved that the free $k$-group on a $C W$-complex $X$ is itself a $C W$-complex containing $X$ as a subcomplex. It follows, as a corollary, that the free topological group on a countable $C W$-complex is a countable CW-complex.

\section{Introduction}

The work of [5] and [12] shows that if $X$ is a $k$-space such that the cartesian product $X \times X$ is not a $k$-space, then the free topological group $F(X)$ is not a $k$-space. In particular, then, if $X$ is Dowker's CW-complex [2] the free topological group $F(X)$ is a priori not a CWcomplex, since it is not even a $k$-space. However, the cartesian product of two countable CW-complexes is always a countable $C W$-complex. So it would seem more reasonable to ask if the free topological group on a countable $C W$-complex is a countable $C W$-complex. In fact we answer a more general question here; by working wholly in the category of $k$-Hausdorff $k$-spaces we prove that the free $k$-group on any $C W$-complex is itself a $C W$-complex containing $X$ as a subcomplex. As a corollary, we then obtain that the free topological group on a countable $C W$-complex is a countable CW-complex.

This investigation was precipitated by a question of Calder, and complements work of [10], which proved that the free product of topological groups which are countable $C W$-complexes is also a countable $C W$-complex.

Received 18 September 1974. Communicated by Sidney A. Morris. The author is especially grateful to P.J. Higgins for his helpful comments and assistance, and also acknowledges the financial aid of the Science Research Council. 


\section{Results}

Before stating any results, we recall the basic definitions and make some preliminary remarks on $k$-spaces.

A topological space $X$ is a $k$-space if a subset $A$ of $X$ is closed in $X$ whenever $f^{-1}(A)$ is closed in $C$, for each compact Hausdorff space $C$ and each continuous map $f: C \rightarrow X$. There is clearly: a category $k_{x}$ of $k$-spaces and continuous maps, and a functor $k:$ top $x+k_{x}$, from the category of all topological spaces to $k_{x}$, which assigns to each topological space $X$ the $k$-space $k X$ obtained by giving the set $X$ the final topology with respect to all continuous maps $f: C \rightarrow X$ from any compact Hausdorff space to $X, k_{x}$ also has a product $x_{k}$, which where no confusion arises will be written just $x$. A topological space $X$ is $k$-Hausdorff if for each compact Hausdorff space $C$ and each continuous map $f: C \rightarrow X, f(C)$ is closed in $X$. Notice that a $k$-space $X$ is $k$-Hausdorff if and only if the diagonal $\Delta_{X}=\{(x, x): x \in X\}$ is closed in $X \times_{k} X$. Throughout this paper, all spaces considered will be $k$-Hausdorff unless otherwise stated. In particular, a CW-complex is a $k$-Hausdorff space which is a closure finite cell-complex with the weak topology. For further information the reader is referred to $[2,4,6,9$, and $11 \mathrm{~J}$.

A $k$-group is a group object in the category $k_{x}$; that is, a group $G$ whose underlying set is a ( $k$-Hausdorff) $k$-space and whose structure functions $\phi: G \times x_{k} G \rightarrow G, \sigma: G \rightarrow G$ are morphisms in $k_{x}$. The (Graev) free $k$-group $[3,4$, and 11] on a pointed $k$-space $(X, e)$ consists of a k-group $F G(X)$ together with a continuous pointed map $i: X \rightarrow F G(X)$ which is universal for continuous pointed maps from $X$ into $k$-groups; that is, if $f: X \rightarrow H$ is any such map then there is a unique morphism of $k$-groups $f^{*}: F G(X) \rightarrow H$ such that $f^{*} i=f . F G(X)$ is independent of the choice of base point, contains $X$ as a closed subset and is algebraically just the free group on the set $X \backslash\{e\}$.

Our main result is

THEOREM 1. The (Graev) free $k$-group $F G(X)$ on a CW-complex $X$ is itself a CW-complex, and contains $X$ as a subcomplex. 
The proof is given in $\$ 3$.

The (Markov) free $k$-group [4 and 8 ] on a $k$-space $X$ is a $k$-group $F M(X)$ together with a continuous map $i: X \rightarrow F M(X)$ such that if $f: X \rightarrow H$ is any continuous map into a $k$-group $B$ then there is a unique morphism of $k$-groups $f^{*}: F M(X) \rightarrow H$ such that $f^{*} i=f$. By checking universal properties, it is easy to prove that if $X$ is any $k$-space, then there is an isomorphism of $k$-groups $F M(X) \cong F G(X \cup e)$, where $X \cup e$ is the disjoint union of $X$ with a singleton space $\{e\}$. Thus Theorem 1 gives us

COROLLARY 2. The (Markov) free $k$-group $F M(X)$ on a CW-complex $X$ is also a CW-complex, containing $X$ as a subcomplex.

We can now obtain a version of Theorem 1 in the usual topological category by a standard argument. A $k_{\omega}$-space $[1,4,7$, and 1.0$]$ is a Hausdorff topological space $X$ which has a countable covering by compact sets $x_{0} \subseteq X_{1} \subseteq \ldots \subseteq x_{n} \subseteq \ldots$ such that $X$ has the weak topology with. respect to $\left\{x_{n}\right\}_{n \geq 0}$. Examples of $k_{\omega}$-spaces are compact Hausdorff spaces, connected locally compact topological groups, and (most important for our purposes) countable CW-complexes. It is clear that any $k_{\omega}$-space is necessarily a $k$-space.

The (Graev) free topological group $[1,3,4,5,7$, and 11$]$ on a pointed topological space is, of course, defined in a similar way to the (Graev) Pree $k$-group on a pointed $k$-space, and it is routine to deduce from the construction of the (Graev) free $k$-group (cf. [11], Theorem 2, and [4], Chapter III, \$4) that if $X$ is a $k_{\omega}$-space, then the (Graev) free $k$-group $F G(X)$ is also the (Graev) free topological group on $X$. But, in the proof of Theorem 1, we will see that if $X$ is a countable CW-complex then $F G(X)$ is also a countable $C W$-complex; so that Theorem 1 again gives

COROLLARY 3. The (Graev) free topological group $F G(X)$ on a countable CW-complex $X$ is itself a countable CW-complex, and contains $X$ as a subcomplex.

A similar result for (Markov) free topological groups can be deduced from Corollary 3 in the same way as Corollary 2 was deduced from Theorem 1 . 


\section{Proof of Theorem 1}

It is clear that we can choose the base point $e \in X$ to be a $O$-cell without loss of generality. Let $X^{-1}$ be a homeomorphic copy of $X$ with elements $x^{-1}$ for each $x \in X$, and let $\bar{X}$ denote the wedge product $X \vee X^{-1}$. Then by the adjunction theorem for $C W$-complexes $([6], p .62$, Theorem 5.11), the obvious cell-structure induced on $\bar{X}$ by the cellstructure on $X$ makes $\bar{X}$ a CW-complex containing both $X$ and $X^{-1}$ as subcomplexes. Now let $\vec{X}^{n}$ be the product in $k_{x}$ of $n$ copies of $\bar{X}$, and let $Y_{n}=\vec{X}^{n} / R_{n}$, where $R_{n}$ is the equivalence relation generated by $\begin{aligned}\left(x_{1}^{\varepsilon_{1}}, \ldots, x_{i-2}^{\varepsilon_{i-2}}, x_{i-1}^{\varepsilon_{i-1}}\right. & \left., x_{i}^{\varepsilon_{i}}, x_{i+1}^{\varepsilon_{i+1}}, x_{i+2}^{\varepsilon_{i+2}}, \ldots, x_{n}^{\varepsilon_{n}}\right) \\ & \sim\left(x_{1}^{\varepsilon_{1}}, \ldots, x_{i-2}^{\varepsilon_{i-2}}, x_{i-1}^{\varepsilon_{i-1}}, e, e, x_{i+2}^{\varepsilon_{i+2}}, \ldots, x_{n}^{\varepsilon_{n}}\right) \\ & \sim\left(x_{1}^{\varepsilon_{1}}, \ldots, x_{i-2}^{\varepsilon_{i-2}}, e, e, x_{i-1}^{\varepsilon_{i-1}}, x_{i+2}^{\varepsilon_{i+2}}, \ldots, x_{n}^{\varepsilon_{n}}\right)\end{aligned}$ whenever $x_{i+1}^{\varepsilon_{i+1}}=x_{i}^{-\varepsilon_{i}}, 1 \leq i<n$. Finally, let $G_{n}$ be the subset of $F G(X)$ comprising all the "reduced words" of length at most $n$ (that is, words $x_{1}^{\varepsilon_{1}}, \ldots, x_{n}^{\varepsilon_{n}}$ in $F G(X)$ such that $x_{i+1}^{\varepsilon_{i+1}} \neq x_{i}^{-\varepsilon_{i}}$ for any $1 \leq i<n$, and $x_{i} \neq e$ for any $\left.1 \leq i \leq n\right)$. Then it is proved in [11], Corollary I (cf. also [4], Chapter V, Theorem 3.1) that each $G_{n}$ is closed in $F G(X)$, and $F G(X)$ is the iterated adjunction space $G_{1} \subseteq G_{2} \subseteq \ldots \subseteq G_{n} \subseteq \ldots$, with $G_{n}=G_{n-1} \cup_{f_{n-1}} y_{n}$, where the attaching $\operatorname{map} f_{n-1}: A_{n-1} \rightarrow G_{n-1}$ is given by $\left(x_{1}^{\varepsilon_{1}}, \ldots, x_{n}^{\varepsilon_{n}}\right)+x_{1}^{\varepsilon_{1}} \ldots x_{n}^{\varepsilon_{n}}$, and $A_{n-1}$ is the subspace of $y_{n}$ consisting of all words $\left(x_{1}^{\varepsilon_{1}}, \ldots, x_{n}^{\varepsilon_{n}}\right)$ which have an " $e$ " somewhere.

Thus to prove Theorem $I$ it is sufficient to prove that each $G_{n}$ is a $C W$-complex containing $G_{n-1}$ as a subcomplex. This we do by induction. 
First we observe that $G_{1}=\bar{X}=X \vee X^{-1}$ is a $C W$-complex. Then for the inductive step, we assume that $G_{n-1}$ is a $C W$-complex; so that, again by the adjunction theorem for $C W$-complexes, it remains only to prove

PROPOSITION 4. For each $n>1$, the space $Y_{n}$ is a CW-complex containing $A_{n-1}$ as a subcomplex.

We will need the following lenma. Let $I^{m}$ be the closed unit $m$-cube in $R^{m}$, and let $S_{m}$ be the equivalence relation generated on $I^{m}$ by

$$
\begin{aligned}
\left(t_{1}, \ldots, t_{i-2}, t_{i-1}, t_{i}, t_{i+1}\right. & \left., t_{i+2}, \ldots, t_{m}\right) \\
& \sim\left(t_{1}, \ldots, t_{i-2}, t_{i-1}, 0,0, t_{i+2}, \ldots, t_{m}\right) \\
& \sim\left(t_{1}, \ldots, t_{i-2}, 0,0, t_{i-1}, t_{i+2}, \ldots, t_{m}\right)
\end{aligned}
$$

whenever $t_{i}=t_{i+1}, 1 \leq i<n$. Then

LEMMA 5. There is a cellular decomposition of $I^{m}$ such that $S_{m}$ is a cellular equivalence relation (cf. [6], p. 32).

Proof. Before describing the cellular decomposition of $I^{m}$, we introduce some new notation. The intersection of a p-cell $P$ with a $q$-cell $Q, p \leq q$, will be called an embeddable intersection if $P \cap Q$ is also a $p$-cell, and will be called a non-degenerate intersection if $P \cap Q$ is a $(p-1)$-cell. Of course an embeddable intersection is necessarily degenerate.

For each $1 \leq i, j \leq m, i \neq j$, let $L_{i j}$ denote the hyperplane $\left\{\left(x_{1}, \ldots, x_{m}\right): x_{i}=x_{j}\right\}$ in $I^{m}$. Then the m-cells of $I^{m}$ are the $m !$ portions into which the $L_{i j}$ divide $I^{m}$.

Now let $M_{i}=\left\{\left(x_{1}, \ldots, x_{m}\right\}: x_{i}=0\right\}$ and $N_{i}=\left\{\left(x_{1}, \ldots, x_{m}\right): x_{i}=1\right\}, 1 \leq i \leq m$, be the faces of $I^{m}$; so that in the "usual" decomposition of $I^{m}$ the $M_{i}, N_{i}$ are precisely the $(m-1)$-cells. Then the (m-1)-cells in our "new" decomposition are all the 
embeddable intersections of the $L_{i j}, M_{k}, N_{l}$ with the m-cells.

Notice that the $(m-2)$-cells in the usual decomposition of $I^{m}$ are just the faces of the $(m-1)$-cells, namely all the non-degenerate intersections of the $M_{i}$ and $N_{j}$. Similarly, in the new decomposition of $I^{m}$, the (m-2)-cells are all the non-degenerate intersections of the $L_{i j}, M_{k}, N_{l}$ with the $(m-1)$-cells. We can now proceed inductively constructing the $(m-r)$-cells of the new decomposition of $I^{m}$ as all the non-degenerate intersections of $L_{i j}, M_{k}, N_{l}$ with the $(n-r+1)$-cells.

The 0 -cells in this decomoosition of $I^{m}$ are of course the same as in the usual decomposition; that is, the "corners" of $I^{m}$.

It is obvious that $I^{m}$ with the above cell-structure is a $C W$-complex, and routine to verify that the equivalence relation $S_{m}$ is a cellular equivalence relation with respect to this cell-structure.

Proof of Proposition 4. First we construct a cell-structure for $Y_{n}$. Let $\bar{X}$ have the cell-structure described above, so that $\phi: \bar{X}+\bar{X}$, given by $x \rightarrow x^{-1}$ and $x^{-1} \rightarrow x$, is a regular homeomorphism. Then for any $m$-cell $\bar{\sigma}: I^{m} \rightarrow \bar{X}$, the composite $I^{m} \stackrel{\bar{\sigma}}{\longrightarrow} \bar{X} \stackrel{\Phi}{\rightarrow}$ is also an $m$-cell for $\bar{X}$, which by an abuse of notation we denote $\bar{\sigma}^{-1}: I^{m} \rightarrow \bar{X}$.

Let $\bar{\sigma}_{i}: I_{i}^{m} \rightarrow \bar{n}_{n}, \quad l \leq i \leq n$, be any $m_{i}$-cells of $\bar{X}$. If $\bar{\sigma}_{i+1} \neq \bar{\sigma}_{i}^{-1}$ for any $1 \leq i<n$, then we have a diagram

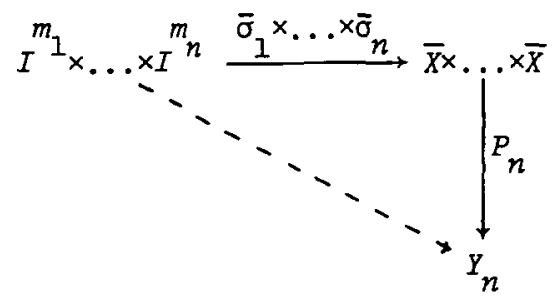

in which $P_{n}: \vec{X}^{n}+y_{n}$ is the canonical quotient map associated with 
$R_{n}$; and so $\sigma=p\left(\bar{\sigma}_{1} \times \ldots \times \bar{\sigma}_{n}\right): I^{m} \times \ldots \times I^{m} \rightarrow y_{n}$ is an $\left(m_{1}+\ldots+m_{n}\right)$-cell for $Y_{n}$. Conversely, if $\bar{\sigma}_{i+1}=\bar{\sigma}_{i}^{-1}$ for some $1 \leq i<n$, then we have a diagram,

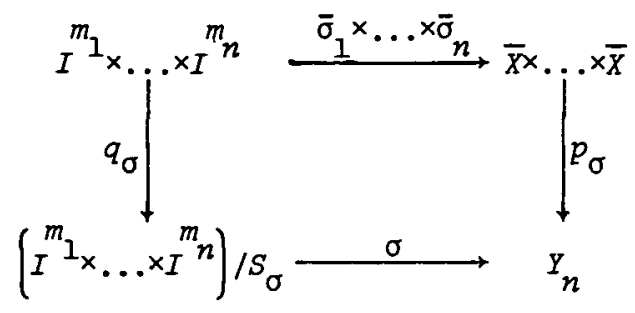

where $S_{\sigma}$ is the equivalence relation generated by

$$
\begin{aligned}
\left(t_{1}, \ldots, t_{i-2}, t_{i-1}, t_{i}, t_{i+1}\right. & \left., t_{i+2}, \ldots, t_{n}\right) \\
& \sim\left(t_{i}, \ldots, t_{i-1}, t_{i}, 0,0, t_{i+2}, \ldots, t_{n}\right) \\
& \sim\left(t_{i}, \ldots, t_{i-2}, 0,0, t_{i-1}, t_{i+2}, \ldots, t_{n}\right)
\end{aligned}
$$

whenever $\bar{\sigma}_{i+1}=\bar{\sigma}_{i}^{-1}$ and $t_{i+1}=t_{i}, 1 \leq i<n$, and $\sigma:\left(I^{m} \times \ldots \times I^{m}\right) / S_{\sigma}+y_{n}$ is the unique map induced by $p_{n}\left(\bar{\sigma}_{1} \times \ldots \times \bar{\sigma}_{n}\right): I^{m} 1 \ldots \times I^{m}+y_{n}$. But by Lemma $5, s_{\sigma}$ is a cellular equivalence relation, so that $\left(I^{m} 1 \times \ldots \times I^{m}\right) / S_{\sigma}$ is a (finite) cell-complex, and $\sigma:\left(I^{m} \times \ldots \times I^{m}\right) / S_{\sigma} \rightarrow Y_{n}$ determines a (finite) number of cells for $Y_{n}$. It is straightforward to check that the set of all cells $\sigma$ constructed as above defines a closure finite cell structure for $Y_{n}$. Thus the proof is completed by

LEMMA 6 . Let $X$ be any $k$-Hausdorff $k$-space having the weak topology with respect to some cover $\left\{x_{\alpha}\right\}$, and let $R$ be an equivalence relation on $X$ such that the groph of $R$ is closed in $X \times X$. Then the quotient space $X / R$ is a $k$-Bausdorff $k$-space having the weak topology with respect to $\left\{x_{\alpha} / R\right\}$. 
The proof is routine (cf. [9], Proposition 2.4).

\section{References}

[1] R. Brown and J.P.L. Hardy, "Subgroups of free topological groups and free topological products of topological groups", J. London Math. Soc. (to appear).

[2] C.H. Dowker, "Topology of metric complexes", Amer. J. Math. 74 (1952), 555-577.

[3] M.1. Graev, "Free topological groups", Amer. Math. Soc. Trons I. (1) 8 (1962), 305-364.

[4] J.P.L. Hardy, "Topological groupoids: coverings and universal constructions", ( $\mathrm{PhD}$ thesis, University College of North Wales, Bangor, 19.74).

[5] J.P.L. Hardy, Sidney A. Morris and H.B. Thompson, "Applications of the Stone-Čech compactification to iree topological groups", submitted.

[6] Albert T. Lundell and Stephen Weingram, The topology of $\mathrm{CW}$ complexes (The University Series in Higher Mathematics, 8. Van Nostrand Reinhold, New York, 1969).

[7] John Mack, Sidney A. Morris and Edward T. Ordman, "Free topological groups and the projective dimension of a locally compact abelian group", Proc. Amer. Math. Soc. 40 (1973), 303-308.

[8] A.A. Markov, "On free topological groups", Amer. Math. Soc. TransZ. (1) $8(1962), 195-272$.

[9] M.C. McCord, "Classifying spaces and infinite symetric products", Trans. Amer. Math. Soc. 146 (1969), 273-298.

[10] Edward T. Ordman, "Free products of topological groups which are $k_{\omega}$-spaces", Trans. Amer. Math. Soc. (to appear).

[11] Edward T. Ordman, "Free k-groups and free topological groups", General Topology and Appl. (to appear). 
[12] Barbara V. Smith Thomas, "Free topological groups", General Topology and Appl. 4 (1974), 51-72.

School of Mathematics and Computer Science,

University College of North Wales,

Bangor,

Gwynedd,

UK. 